\begin{tabular}{c} 
International Journal of Physical Research, 4 (1) (2016) $15-19$ \\
International Journal of Physical Research \\
WPC \\
Website: $\begin{array}{c}\text { ww.sciencepubco.com/index.php/IJPR } \\
\text { doi: } 10.14419 / \text { ijpr.v4il.5902 } \\
\text { Research paper }\end{array}$ \\
\hline
\end{tabular}

\title{
Inhomogeneous cosmology with quasi-vacuum effective equation of state on Lyra manifold
}

\author{
V. K. Shchigolev ${ }^{1 *}$ \\ ${ }^{1}$ Department of Theoretical Physics, Ulyanovsk State University, Ulyanovsk, 432000, Russia \\ *Corresponding author E-mail:vkshch@yahoo.com
}

\begin{abstract}
A class of inhomogeneous Lemaître-Tolman cosmological models is obtained in the context of Lyra's geometry. Cosmological models in Lyra's geometry are studied under the condition of the minimal coupling of matter with the displacement vector field and the varying $\Lambda$ term. Exact solutions to the model equations are obtained subject to the quasi-vacuum effective equation of state. As a result, the displacement field as well as the cosmological term can be expressed in terms of the energy density of matter. The rate of expansion and the deceleration parameter of the model are also studied.
\end{abstract}

Keywords: Cosmology; Lemaître-Tolman metrics; Lyra geometry.

\section{Introduction}

Recent observations like type Ia Supernovae [1, 2], the cosmic microwave background radiation [3], and the large-scale structure [4] strongly indicate that our universe is spatially flat and currently undergoing an accelerating expansion. This acceleration could be realized with negative pressure and positive energy density of some exotic matter commonly called "dark energy" (DE). Due to the DE repulsive effect, the universe has experienced the transition from the earlier deceleration phase to the recent acceleration phase at some instant in the past [5]. Unfortunately, the source of accelerated expansion is still unknown. In physical cosmology and astronomy, the simplest candidate for the dark energy is the cosmological constant $\Lambda$, but it needs to be extremely fine-tuned to satisfy the current value of the DE density. Alternatively, to explain the decay of this density, the different forms of dynamically changing DE with an effective equation of state $(\mathrm{EoS}) w_{e f f}=p_{\text {eff }} / \rho_{\text {eff }}<-1 / 3$ were proposed instead of the constant vacuum energy density $w_{v a c}=-1$.

Another approach to the explanation of accelerated expansion consists of different modifications of the gravitational theory itself. Several theories are proposed as alternatives to Einstein's theory to reveal the nature of the universe in the early stage of evolution. The most attractive among them were scalar-tensor theories proposed by Brans and Dicke [6], Lyra [7], Nordtvedt [8], Saez and Ballester [9], Barber [10] and some others.

G. Lyra [7] suggested a modification of Riemannian geometry by introducing a gauge function into the structureless manifold, as a result of which the cosmological constant arises naturally from the geometry. Sen and Dunn [11] have proposed a new scalar tensor theory of gravitation and constructed an analogue of the Einstein field equation based on Lyra's geometry. According to Halford [12] the scalar-tensor treatment based on Lyra's geometry predicts the same effects within observational limits as in Einstein's theory.
Soleng [13] has pointed out that the constant displacement field in Lyra's geometry will either include a creation field and be equal to Hoyle's creation field cosmology or contain a special vacuum field, which together with the gauge vector form may be considered as a cosmological term. Subsequent investigations were done by several authors in cosmology within the frame work of Lyra's geometry (see, e.g., some latest papers [14]-[22]).

Inhomogeneous cosmological models are those exact solutions of Einstein's equations that contain at least a subclass of non-vacuum, non-static Friedmann-Robertson-Walker (FRW) solutions as a limit. Inhomogeneous models of the universe arise from perturbations of the FRW metric. The Lemaitre-Tolman (LT) models are one of the oldest solutions in general relativity that describe spherically symmetric inhomogeneous non-dissipative fluid. The cosmological models describing a spherically symmetric inhomogeneous universe in General Relativity filled with dust matter without pressure, possibly including the cosmological constant, were originally discussed by Lemaître [23] and Tolman [24] and have been widely studied. Apart from the study of gravitational collapse, these models have also been used in the study of quantum gravity, cosmology and also the inhomogeneities in the universe which may account for the present accelerated expansion of the universe.

Recently the LT model has been used to explain the cosmological data: it represents an alternative to the introduction of the notion of dark energy in the standard cosmological model. For a comprehensive exposition of the LT models and subsequent developments one can refer to [25, 26]. As noted in [27], instead of invoking exotic energy sources, it could be suggested that the cosmic acceleration might originate from the inhomogeneities of the universe. The possible change of the deceleration parameter in an inhomogeneous universe has been studied in [28].

At the same time, the observational data and our knowledge presume the paradigm of cosmological inflation regarding the very early 
universe. Along with all kinds of inhomogeneous and anisotropic scenarios of inflation, the author of this work investigated earlier the possibility of cosmological inflation within the framework of LT model in [29].

Thus, it would be worthwhile to explore the LT models in the context of modified theories of gravity. In this paper, we consider minimally interacting perfect fluid and displacement field with a varying cosmological term within the framework of a LT space-time in Lyra's geometry. Some exact solutions to the model equations are obtained subject to the quasi-vacuum effective equation of state. We show that the displacement field the varying cosmological term in our model can be expressed in terms of the energy density of matter. We also consider the rate of expansion and the deceleration parameter of the model.

\section{Lyra's geometry in brief}

Let us briefly recall the main ideas of Lyra's geometry [7]. It is useful to mention that Lyra's geometry can be considered as a generalization of Riemannian geometry by introducing a gauge function which removes the non-integrability of the length of the parallel transfer characteristic of Weyl's theory. In Lyra's geometry, the displacement vector between two neighboring points $x^{i}$ and $x^{i}+d x^{i}$ is determined by the components $\Psi d x^{i}$, where $\Psi=\Psi\left(x^{k}\right)$ is a gauge function. A reference frame $\left(\Psi, x^{i}\right)$ consists of the coordinate system $x^{i}$ and the gauge function $\Psi$. Therefore, the transition to the new reference frame $\left(\Psi^{\prime}, x^{\prime i}\right)$ is given by

$\Psi^{\prime}=\Psi\left(\Psi, x^{k}\right), \quad x^{\prime i}=x^{i}\left(x^{k}\right)$,

where $\partial \Psi^{\prime} / \partial \Psi \neq 0, \operatorname{det}\left|\partial x^{\prime i} / \partial x^{k}\right| \neq 0$. The Levi-Civita connection coefficients in Lyra geometry are defined as follows:

$* \Gamma_{j k}^{i}=\Psi^{-1} \Gamma_{j k}^{i}-\frac{1}{2}\left(\delta_{j}^{i} \phi_{k}+\delta_{k}^{i} \phi_{j}-g_{j k} \phi^{i}\right)$,

where $\Gamma_{j k}^{i}$ is defined in terms of the metric tensor $g_{i k}$ just like as in Riemannian geometry, and $\phi_{k}$ is the displacement vector field. Lyra [7] and Sen and Dunn [11] have showed that any general frame of reference vector field $\phi_{k}$ arises as a natural consequence of the introduction of the gauge function $\Psi$ in the structureless manifold. The metric on the Lyra manifold is determined by the interval

$d s^{2}=\Psi^{2} g_{i k} d x^{i} d x^{k}$,

which is invariant with respect to the coordinate and gauge transformations.

As a result, the parallel transport of a vector $\xi^{i}$ is given by

$d \xi^{i}=-\tilde{\Gamma}_{j k}^{i} \xi^{j} \Psi d x^{k}$,

where

$\tilde{\Gamma}_{j k}^{i}=* \Gamma_{j k}^{i}-\frac{1}{2} \delta_{j}^{i} \phi_{k}$.

Thus, we see that $\tilde{\Gamma}^{i}{ }_{j k}$ is not symmetric with respect to $j$ and $k$. A remarkable difference from the Weyl geometry is that in Lyra geometry length of the vector does not change under parallel transport.

As always, the curvature tensor is defined in terms of the parallel transport of a vector along a closed curve, and is equal to

$* R_{. j k l}^{i}=\Psi^{-2}\left[-\left(\Psi \tilde{\Gamma}_{j k}^{i}\right)_{, l}+\left(\Psi \tilde{\Gamma}_{j l}^{i}\right)_{, k}-\Psi^{2}\left(\tilde{\Gamma}_{j k}^{m} \tilde{\Gamma}_{m l}^{i}-\tilde{\Gamma}_{j l}^{m} \tilde{\Gamma}_{k m}^{i}\right)\right]$,

where $\tilde{\Gamma}_{j k}^{i}$ is determined from (5). The convolution of the curvature tensor (6) yields the scalar curvature

$* R=\Psi^{-2} R+3 \Psi^{-1} \phi_{; i}^{i}+\frac{3}{2} \phi^{i} \phi_{i}+2 \Psi^{-1}\left(\ln \Psi^{2}\right)_{, i} \phi^{i}$,

where $R$ is the Riemannian scalar of curvature, and the semicolon denotes the covariant derivative with the Christoffel symbols of the second kind.
The action integral is invariant under the gauge and coordinate transformations, and is given in the form

$S=\int \mathscr{L} \sqrt{-g} \Psi^{4} d^{4} x$

where $d^{4} x$ is a volume element, and $\mathscr{L}$ is a scalar function.

Using the normal gauge $\Psi=1$ [11], and putting $\mathscr{L}=* R$ in equation (8), it is easy to find that equation (7) can be reduced to the following form

$* R=R+3 \phi_{; i}^{i}+\frac{3}{2} \phi^{i} \phi_{i}$.

The field equation is obtained from the variational principle

$\delta\left(S+S_{m}\right)=0$,

where $S$ is defined by (8), and the action $S_{m}$ is defined by the Lagrangian density of matter $\mathscr{L}_{m}$ as usual:

$S_{m}=\int \mathscr{L}_{m} \sqrt{-g} d^{4} x$.

\section{The model equations}

The Einstein's field equations in Lyra's geometry, proposed in [11] in normal gauge, can be written with a variable $\Lambda$ - term as

$R_{i k}-\frac{1}{2} g_{i k} R-\Lambda g_{i k}+\frac{3}{2} \phi_{i} \phi_{k}-\frac{3}{4} g_{i k} \phi^{j} \phi_{j}=T_{i k}$,

where $\phi_{i}$ is a displacement vector. For simplicity, we assume that the gravitational constant $8 \pi G=1$. All other symbols have their usual meanings in the Riemannian geometry.

The energy-momentum tensor (EMT) of matter $T_{i k}^{(m)}$ can be derived in a usual manner from the Lagrangian of matter (11). Considering the matter as a perfect fluid, we have

$T_{i k}^{(m)}=\left(\rho_{m}+p_{m}\right) u_{i} u_{k}-p_{m} g_{i k}$,

where $u_{i}=(1,0,0,0)$ is 4-velocity of the co-moving observer, satisfying $u_{i} u^{i}=1, \rho_{m}$ and $p_{m}$ are the energy density and pressure of matter, consequently.

In cosmological studies on the Lyra manifold, the contribution of the displacement field in Einstein's equation (12) is often represented by the energy-momentum tensor

$T_{i k}^{(\phi)}=-\frac{3}{2} \phi_{i} \phi_{k}+\frac{3}{4} g_{i k} \phi^{j} \phi_{j}$,

despite of its purely geometric nature. At the same time, the energymomentum tensor of a cosmological term can be represented by

$T_{i k}^{(\Lambda)}=\Lambda g_{i k}$

As a result, we can represent the Einstein equation (12) in the following simple form

$G_{i k}=\widetilde{T}_{i k} \equiv T_{i k}^{(m)}+T_{i k}^{(\phi)}+T_{i k}^{(\Lambda)}$

by introducing the effective EMT $\widetilde{T}_{i k}$, and the Einstein tensor $G_{i k}=$ $R_{i k}-(1 / 2) g_{i k} R$.

Let $\phi_{i}$ be a time-like vector field of displacement,

$\phi_{i}=\left[\frac{2}{\sqrt{3}} \beta(r, t), 0,0,0\right]$,

where the numerical factor $2 / \sqrt{3}$ is substituted for the sake of convenience in what follows. 
Let us assume that dynamics of a spherically symmetric universe is described by the LT solution to Einstein's field equations described by the well-known LT metrics $[23,24]$

$d s^{2}=d t^{2}-\frac{\left(R^{\prime}\right)^{2}}{f^{2}} d r^{2}-R^{2}\left(d^{2} \theta+\sin ^{2} \theta d^{2} \varphi\right)$,

where $R=R(r, t)>0$ is a function to be determined. In the case $R(r, t)=a(t) g(r), f(r)=g^{\prime}(r), g(r)=\{\sin (r), r, \sinh (r)\}$, the LT metrics is identical to a homogeneous FRW metric. Otherwise, the LT metrics corresponds to the inhomogeneous cosmological model, but still remains isotropic around one observer. Hereafter, the prime denotes a derivative with respect to $r$, while the over-dot stands for the time derivative.

For the LT space-time interval (15), the Einstein equation (16) can be written down in the following form

$\frac{F^{\prime}}{2 R^{\prime} R^{2}}=\widetilde{T}_{0}^{0}, \quad \frac{\dot{F}}{2 \dot{R} R^{2}}=\widetilde{T}_{1}^{1}, \quad \frac{1}{4 R^{\prime} R}\left(\frac{\dot{F}}{\dot{R}}\right)^{\prime}=\widetilde{T}_{2}^{2}=\widetilde{T}_{3}^{3}$,

where the so-called "mass function" is given by

$F(r, t)=2 R \dot{R}^{2}+2 R\left(1-f^{2}\right)$

Combining Einstein's equations in (15), one can derive the following equations of the energy-momentum conservation law

$\widetilde{T}_{2}^{2}=\widetilde{T}_{1}^{1}+\frac{R}{2 R^{\prime}}\left(\widetilde{T}_{1}^{1}\right)^{\prime}$

$R^{2}\left[\left(\dot{T}_{0}^{0}\right) R^{\prime}-\left(\widetilde{T}_{1}^{1}\right)^{\prime} \dot{R}\right]+\left(\widetilde{T}_{0}^{0}-\widetilde{T}_{1}^{1}\right) \frac{\partial\left(R^{2} R^{\prime}\right)}{\partial t}=0$,

which also could be obtained from the conservation law, $\widetilde{T}_{i ; l}^{j}=0$, for the effective EMT in the LT metrics (15).

\section{Exact solutions to the field equations}

From equations (13)-(17), the following expressions for the non-zero components of effective EMT can be obtained

$$
\begin{aligned}
\widetilde{T}_{0}^{0} & =\rho_{\text {eff }}(r, t)=\rho_{m}+\beta^{2}+\Lambda, \\
\widetilde{T}_{1}{ }^{1} & =\widetilde{T}_{2}^{2}=\widetilde{T}_{3}^{3}=-p_{e f f}(r, t)=-p_{m}-\beta^{2}+\Lambda .
\end{aligned}
$$

Applying these equations to the conservation law (21), (22), we get $\left(p_{e f f}\right)^{\prime}=0$, i.e. $p_{e f f}=p_{e f f}(t)$, and

$\frac{\partial \rho_{e f f}}{\partial t}+\left(\rho_{e f f}+p_{e f f}\right) \frac{\partial \ln \left(R^{2} R^{\prime}\right)}{\partial t}=0$.

Suppose further that the effective energy density and pressure satisfy the effective EoS of the following form

$p_{e f f}=w_{e f f} \rho_{e f f}$,

where the effective barotropic index $w_{\text {eff }}$ is in general a function of $r$ and $t$. However, let us consider the case of quasi-vacuum effective EoS, i.e. $w_{e f f}=-1$. Substituting $p_{e f f}=-\rho_{e f f}$ in equation (24), one get $\dot{\rho}_{e f f}=0$, or $\rho_{e f f}=\rho_{e f f}(r)$. It is obvious now that the equality in (25) for $w_{e f f}=-1$ is possible only if both sides of this equation are constant. Let us denote this constant $\Lambda_{0}$. Therefore, we have

$\rho_{\text {eff }}=-p_{\text {eff }}=\Lambda_{0}$.

Taking into account (19), (20), (23) and (26), one can obtain the partial derivatives equation of the first order for $R(r, t)$ as follows

$R\left(\frac{\partial R}{\partial t}\right)^{2}+R\left(1-f^{2}\right)=\frac{\Lambda_{0}}{3}\left(R^{3}+\delta R_{0}^{3}\right)$, where $R_{0}$ is a constant of integration, and $\delta=0, \pm 1$. This equation can be readily integrated (see, e.g., [26, 29] and references therein). In the case of the parabolic model, i.e. under the condition $f^{2}=1$, the result of solving equation (28) can be represented as follows

$$
R_{\delta}^{p}(r, t)= \begin{cases}R_{0} \sinh ^{2 / 3}\left[\frac{3}{2} \sqrt{\frac{\Lambda_{0}}{3}}\left(t+t_{0}(r)\right)\right], & \delta=+1, \\ R_{0} r \exp \left(\sqrt{\frac{\Lambda_{0}}{3}} t\right), & \delta=0, \\ R_{0} \cosh ^{2 / 3}\left[\frac{3}{2} \sqrt{\frac{\Lambda_{0}}{3}}\left(t+t_{0}(r)\right)\right], & \delta=-1\end{cases}
$$

where $R_{0}$ is a constant, and $t_{0}(r)$ is an arbitrary differentiable function specifying the instant of the Big Bang, which is seen to be position-dependent. As one can see, an appropriate rescaling has been chosen in the case of $\delta=0$ to satisfy the gauge $R(0, r)=R_{0} r$ [30].

For the hyperbolic, $f^{2}>1$, and the elliptical, $f^{2}<1$, models, it is easy to find solutions for equation (26) with $\delta=0$ in the following form

$$
R_{0}^{h, e}(r, t)=\left\{\begin{array}{l}
\sqrt{\frac{3\left(f^{2}-1\right)}{\Lambda_{0}}} \sinh \left[\sqrt{\frac{\Lambda_{0}}{3}}\left(t+t_{0}(r)\right)\right] ; f^{2}>1, \\
\sqrt{\frac{3\left(1-f^{2}\right)}{\Lambda_{0}}} \cosh \left[\sqrt{\frac{\Lambda_{0}}{3}}\left(t+t_{0}(r)\right)\right] ; f^{2}<1 .
\end{array}\right.
$$

In the case $\delta \neq 0$, equation (27) for $R_{\delta}^{h, e}(r, t)$ can be solved in terms of the Weierstrass elliptic function [23].

From equations (23) and (26), it follows that

$\rho_{m}+\beta^{2}+\Lambda=\Lambda_{0}, \quad p_{m}+\beta^{2}-\Lambda=-\Lambda_{0}$.

It is interesting to note that all dynamical parameters in these equations can arbitrarily depend on $t$ and $r$, as there are no other restrictions on them. Indeed, the main equations such as Einstein's equation (19) and the continuity equations (21), (22) are already satisfied by (26). Assuming the matter energy density and pressure satisfy the barotropic $\operatorname{EoS} p_{m}=w_{m} \rho_{m}$ in (30), we are able to express the displacement field and the cosmological term by means of the matter density as follows

$\beta^{2}=-\frac{1}{2}\left(1+w_{m}\right) \rho_{m}, \quad \Lambda=\Lambda_{0}-\frac{1}{2}\left(1-w_{m}\right) \rho_{m}$

Hence it is clear that the matter density in this model should be represented by a phantom matter in its nature (that is $w_{m}<-1$ ), as the displacement field is a real function $\left(\beta^{2}>0\right)$. Thus, we can hitherto observe considerable arbitrariness in the behavior of matter. The requirement of a minimum matter coupling to the displacement vector and the cosmological term can significantly limit this arbitrariness. This means that equation (25) breaks up into two independent equations

$\frac{\partial \rho_{m}}{\partial t}+\left(1+w_{m}\right) \rho_{m} \frac{\partial \ln \left(R^{2} R^{\prime}\right)}{\partial t}=0$,

and

$\frac{\partial \beta^{2}}{\partial t}+2 \beta^{2} \frac{\partial \ln \left(R^{2} R^{\prime}\right)}{\partial t}=-\frac{\partial \Lambda}{\partial t}$.

despite that equations (30) are valid so far. Therefore, we could solve equation (32), and then substitute $\rho_{m}$ obtained into (31). Equation (32) is readily integrated for a constant EoS of matter, $w_{m}=$ constant, that yields

$\rho_{m}(r, t)=\mu(r)\left(R^{2} R^{\prime}\right)^{-\left(1+w_{m}\right)}$,

where $\mu(r)$ is an arbitrary differentiable function, which defines along with $t_{0}(r)$ the mass density distribution at some instant. 


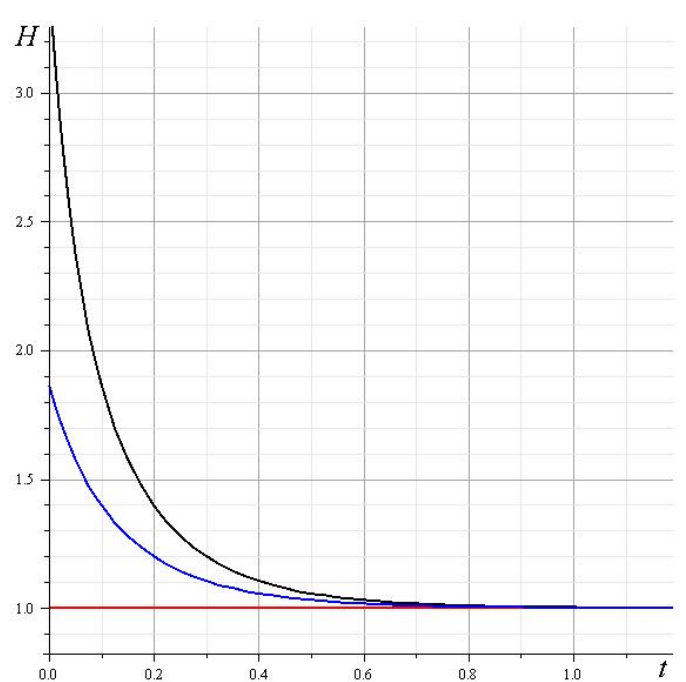

Figure 1: The Hubble parameter $H_{\delta}^{p}$ versus time $t$ according to (36) with $\delta=0$ (red line), and $\delta= \pm 1$ for $t_{0}=0.1$ (black line) and $t_{0}=0.2$ (blue line).

Let us consider some properties of our model. As it emphasized in [30], for inhomogeneous model the directional preference need to be specified in the expression for expansion. With the help of a projection tensor, one can define the invariant expansion rates in two perpendicular directions as

$H_{r}=\frac{\dot{R}^{\prime}}{R^{\prime}}, \quad H_{\perp}=\frac{\dot{R}}{R}$,

so that the average expansion rate is given by

$H=\frac{2}{3} H_{\perp}+\frac{1}{3} H_{r}$.

Using, for example, $R_{\delta}^{p}(r, t)$ for the parabolic model from equation (28) in (34) and (35), we can obtain the following expansion rate for this case

$$
H_{\delta}^{p}(r, t)= \begin{cases}\sqrt{\frac{\Lambda_{0}}{3}}, & \delta=0 \\ \sqrt{\frac{\Lambda_{0}}{3}} \operatorname{coth}\left[3 \sqrt{\frac{\Lambda_{0}}{3}}\left(t+t_{0}(r)\right)\right], & \delta= \pm 1 .\end{cases}
$$

The behavior of the average Hubble parameter (36) with time for different values of $\delta$ and $t_{0}(r)$ is shown in Fig. 1. To be specific, we have chosen the units in which $\sqrt{\Lambda_{0} / 3}=1$. From these graphs, it is clear that the expansion rate increases for greater $t_{0}(r)$ i.e., greater inhomogeneity.

It should be noted that the introduction of the Hubble parameter as well as the deceleration parameter in the LT model is ambiguous. Considering the model with zero pressure and zero cosmological constant, J. Moffat [28] proposed the effective Hubble parameter $H_{e f f}^{2}=H_{\perp}^{2}+2 H_{\perp} H_{r}$, where $H_{\perp}$ and $H_{r}$ are determined by (34), and essentially differs from (35). However, the model-independent expression for the deceleration parameter $q$ has been derived in [28] by expanding $R(r, t)$ in a Taylor series

$R(r, t)=R\left(r, t_{p}\right)\left[1-\left(t_{p}-t\right) H_{p \perp}-\frac{1}{2}\left(t_{p}-t\right)^{2} q\left(r, t_{p}\right) H_{p \perp}^{2}-\ldots\right]$

where $t_{p}$ is the present instant, so that the deceleration parameter is given by

$q(r, t)=-\left(\frac{R}{\dot{R}}\right)^{2} \frac{\ddot{R}}{R}=-\frac{1}{H_{\perp}^{2}} \frac{\ddot{R}}{R}$.

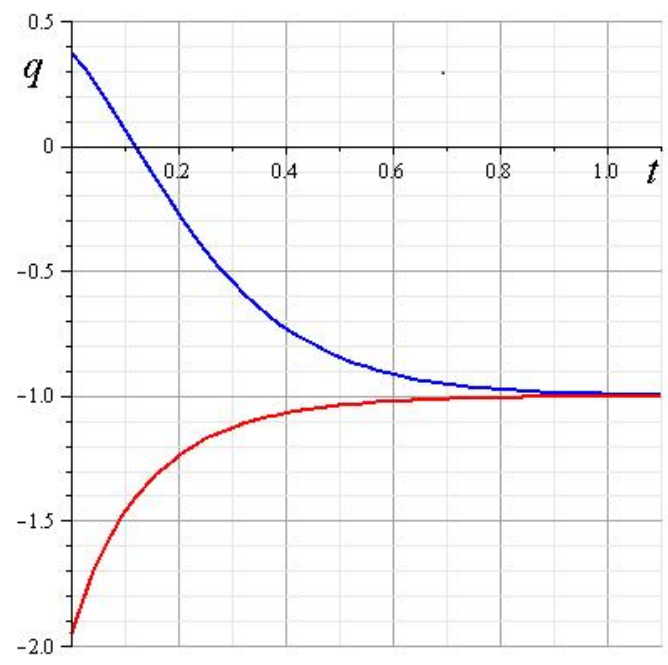

Figure 2: The deceleration parameter $q$ versus time $t$ according to (38) with $\delta=+1, t_{0}=0.1$ (blue line), and $\delta=-1, t_{0}=0.35$ (red line).

Using the same example of $R_{\delta}^{p}(r, t)$ for the parabolic model (28) and (37), one can readily obtain the following deceleration parameter

$q_{\delta}(r, t)= \begin{cases}\frac{1}{2}\left\{1-3 \tanh ^{2}\left[\frac{3}{2} \sqrt{\frac{\Lambda_{0}}{3}}\left(t+t_{0}(r)\right)\right]\right\}, & \delta=+1, \\ -1, & \delta=0, \\ \frac{1}{2}\left\{1-3 \operatorname{coth}^{2}\left[\frac{3}{2} \sqrt{\frac{\Lambda_{0}}{3}}\left(t+t_{0}(r)\right)\right]\right\}, & \delta=-1 .\end{cases}$

Fig. 2 shows that our model experiences eternal accelerated expansion in the cases $\delta=0$ and $\delta=-1$, but the model $\delta=+1$ passes from the deceleration state to the acceleration state at some instant in the past.

\section{Conclusion}

In this paper, we have considered the inhomogeneous LT cosmological model in the context of Lyra geometry. The solutions of the field equations have been obtained and discussed in three different types of spacetime, namely the parabolic, elliptic and hyperbolic models, under the condition of the quasi-vacuum effective EoS.

The most interesting feature of this model is that the EoS of matter can be almost arbitrary as well as inhomogeneous, even though the effective EoS is fixed and equals -1 . This has become possible as a result of fine-tuning the displacement field and cosmological term, expressed by equation (31). A simple example of solution for the energy density of matter with constant EoS is given by equation (33). At that, we have found that the physical matter should satisfy the phantom EoS that is known to be easily modeled by means of phantom scalar field. For the parabolic model, we have investigated some of its kinematic properties and have illustrated their parameters graphically. Specifically, we have obtained the rate of expansion and the deceleration parameter for the parabolic model given by (36) and (38), respectively.

It is obvious that more detailed properties of our model requires a further study that goes beyond the purpose of the present paper.

\section{References}

[1] A. G. Riess , et al. , "Observational Evidence from Supernovae for an Accelerating Universe and a Cosmological Constant", Astronomical Journal, Vol. 116 (1998), 1009. http://dx.doi.org/10.1086/300499

[2] S. Perlmutter, et al., "Measurements of Omega and Lambda from 42 High-Redshift Supernovae", Astrophysical Journal, Vol. 517 (1999), 565. http://dx.doi.org/10.1086/307221 
[3] N. Jarosik, C.L. Bennett, et al., "Seven-Year Wilkinson Microwave Anisotropy Probe (WMAP) Observations: Sky Maps, Systematic Errors, and Basic Results", Astrophysical Journal Supplement Series, 192 (2011), 14. http://dx.doi.org/10.1088/0067-0049/192/2/14

[4] S. F. Daniel, R. R. Caldwell, A. Cooray and A. Melchiorri, "Large scale structure as a probe of gravitational slip", Physical Review D 77 (2008), 103513. http://dx.doi.org/10.1103/PhysRevD.77.103513

[5] R. R. Caldwell, W. Komp, L. Parker and D. A. T. Vanzella, "Sudden gravitational transition", Physical Review D 73 (2006), 023513. http://dx.doi.org/10.1103/PhysRevD.73.023513

[6] H. Brans, and R. H. Dicke, "March's Principle and a Relativistic Theory of Gravitation", Physical Review A 124 (1961), 925-935. http://dx.doi.org/10.1103/PhysRev.124.925

[7] G. Lyra, "Über eine modifikation der riemannschen geometrie", Mathematische Zeitschrift 54 (1951), 52-64. http://dx.doi.org/10.1007/BF01175135

[8] K. Nordtvedt Jr., "Post-Newtonian Metric for a General Class of Scalar-Tensor Gravitational Theories and Observational Consequences, The Astrophysical Journal 161 (1970), 1059-1067. http://dx.doi.org/10.1086/150607

[9] D. Saez and V.J. Ballester, "A Simple Coupling with Cosmological Implications", Physical Letters A 113 (1986), 467-470. http://dx.doi.org/10.1016/0375-9601(86)90121-0

[10] G.A. Barber, "On Two "Self-Creation" Cosmologies", General Relativity and Gravitation 14 (1982), 117136.http://dx.doi.org/10.1007/s10509-007-9387-x

[11] D. K. Sen and K. A. Dunn, "A Scalar-Tensor Theory of Gravitation in a Modified Riemannian Manifold", Journal of Mathematical Physics 12 (1971), 578. http://dx.doi.org/10.1063/1.1665623

[12] W. D. Halford, "Scalar-tensor theory of gravitation in a Lyra manifold”, Journal of Mathematical Physics 13 (1972), 1699-1703. http://dx.doi.org/10.1063/1.1665894

[13] H. H. Soleng, "Cosmologies based on Lyra's geometry," General Relativity and Gravitation 19 (1987), 1213-1216. http://dx.doi.org/10.1007/BF00759100

[14] V. K. Shchigolev, "Cosmology with an Effective $\Lambda$-term in lyra manifold", Chinese Physics Letters 30 (2013), 119801. doi:10.1088/0256307X/30/11/119801

[15] Hoavo Hova, "A Dark Energy Model in Lyra Manifold", Journal of Geometry and Physics 64 (2013), 146-154. http://dx.doi.org/10.1016/j.geomphys.2012.08.004

[16] Haizhao Zhi, Mengjiao Shi, Xinhe Meng, Lianzhong Zhang "A new global 1-form in Lyra geometric cosmos model", International Journal of Theoretical Physics, Vol.53, Issue 11, (2014), pp 4002-4011. http://dx.doi.org/10.1007/s10773-014-2151-4

[17] M. Khurshudyan, J. Sadeghi, R. Myrzakulov, A. Pasqua, H. Farahani, "Interacting quintessence dark energy models in Lyra manifold", Advances in High Energy Physics 2014 (2014), 878092 http://dx.doi.org/10.1155/2014/878092

[18] V. K. Shchigolev, E. A. Semenova, "Scalar field cosmology in Lyra's geometry", International Journal of Advanced Astronomy, Vol. 3, No. 2 (2015), 117-122. http://dx.doi.org/10.14419/ijaa.v3i2.5401

[19] M. Khurshudyan, "Interacting extended Chaplygin gas cosmology in Lyra manifold", Astrophys and Space Science 360 (2015), 44. http://dx.doi.org/10.1007/s10509-015-2557-3

[20] M. Khurshudyan, A. Pasqua, J. Sadeghi, H. Farahani, ”Quintessence Cosmology with an Effective $\Lambda$-Term in Lyra Manifold", Chinese Physics Letters 32(2015) 109501. http://dx.doi.org/10.1088/0256307X/32/10/109501

[21] M. Khurshudyan, Toy Models of Universe with an Effective Varying $\Lambda$-Term in Lyra Manifold, Advances in High Energy Physics Vol.2015, Article ID 796168, 10 pages. http://dx.doi.org/10.1155/2015/796168

[22] M. Khurshudyan, J. Sadeghi, A. Pasqua, S. Chattopadhyay, R. Myrzakulov, H. Farahani, "Interacting Ricci dark energy models with an effective $\Lambda$-term in Lyra manifold", International Journal of Theoretical Physics 54 (2015), 749. http://dx.doi.org/10.1007/s10773-014-2266-7

[23] G. Lemaître, "L'univers en expansion", Ann. Soc. Sci. Bruxelles A53(1933) 51 ; reproduced as a Golden Oldie and translated by M. A. H. MacCallum, General Relativity and Gravitation 29(5) (1997), 641. http://dx.doi.org/10.1023/A:1018855621348

[24] R.C. Tolman, Effect of Inhomogeneity on Cosmological Models", Proceedings of the National Academy of Sciences 20 (1934), 169 http://www.pnas.org/content/20/3/169.full.pdf

[25] A. Krasinski, Inhomogeneous Cosmological Models, Cambridge University Press, Cambridge, (1997).

[26] Antonio Zeccaa, "Lemaître-Tolman-Bondi model: Solutions of the cosmological equation", European Physical Journal Plus 128 (2013), 107. http://dx.doi.org/10.1140/epjp/i2013-13107-0

[27] Chia-Hsun Chuang, Je-An Gu and W-Y P Hwang, "Inhomogeneityinduced cosmic acceleration in a dust universe", Classical and Quantum Gravity 25 (2008), 175001. http://dx.doi.org/10.1088/02649381/25/17/175001

[28] J. W. Moffat, "Late-time inhomogeneity and acceleration without dark energy", Journal of Cosmology and Astroparticle Physics 05 (2006) 001. http://dx.doi.org/10.1088/1475-7516/2006/05/001

[29] V. K. Shchigolev, Exact inhomogeneous models of cosmological inflation", Russian Physics Journal, Vol.41, Issue 2 (1998), 89-92. http://dx.doi.org/10.1007/BF02766550
[30] D. Panigrahi and S. Chatterjee, "Spherically symmetric inhomogeneous model with Chaplygin gas", arXiv:1108.2433v2 [gr-qc] 